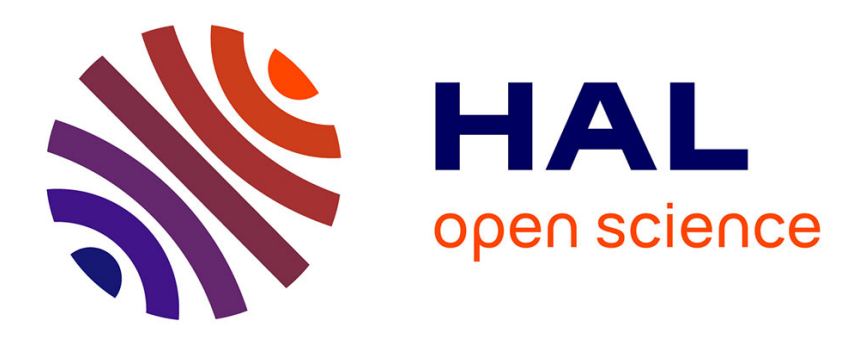

\title{
An explanation of the quantum mechanics of particles Oscar Chavoya Aceves
}

\section{To cite this version:}

Oscar Chavoya Aceves. An explanation of the quantum mechanics of particles. 2014. hal-01054953

\section{HAL Id: hal-01054953 https://hal.science/hal-01054953}

Preprint submitted on 11 Aug 2014

HAL is a multi-disciplinary open access archive for the deposit and dissemination of scientific research documents, whether they are published or not. The documents may come from teaching and research institutions in France or abroad, or from public or private research centers.
L'archive ouverte pluridisciplinaire HAL, est destinée au dépôt et à la diffusion de documents scientifiques de niveau recherche, publiés ou non, émanant des établissements d'enseignement et de recherche français ou étrangers, des laboratoires publics ou privés. 


\title{
An explanation of the quantum mechanics of particles*
}

\author{
O. Chavoya-Aceves \\ Glendale Community College \\ Department of Physical Sciences \\ 6000 W Olive Ave, Glendale, AZ 85302 \\ chavoyao@gmail.com
}

August 11, 2014

\begin{abstract}
The ontological status of the wave function as a purely mathematical artifact is clarified. Grounds are found to reject the de BroglieBohm formulation of the quantum mechanics of particles. The presumption by Einstein-Podolsky-Rosen that quantum mechanics is not a complete theory is reinforced.
\end{abstract}

\section{Introduction}

Despite R. Feynman's warning [1]:

"What I am going to tell you about is what we teach our physics students in the third or fourth year of graduate school - and you think I'm going to explain it to you so you can understand it? No, you are not going to be able to understand it. Why, then, am I going to bother you with all this? Why are you going to sit here all this time, when you won't be able to understand what I say? It is my task to convince you not to turn away because you don't

*Submitted to Physics Essays 
understand. You see, my physics students don't understand it either. That is because $I$ don't understand it. Nobody does."

in this paper we attempt a rational explanation of the principles of the quantum mechanics of particles.

We have taken this challenge because: If it is the case that nobody understands quantum mechanics, then it's also the case that nobody knows if it is true. Our aim is to clarify the formalism and try to separate what is essentially abstract mathematics from what has a physical meaning. In our way to clarify the meaning of wave functions we have found grounds to criticize the de Broglie-Bohm formulation of Quantum Mechanics as described in $[3,5,6]$. Also, in our view, this paper reinforces the Einstein-Podolsy-Rosen presumption that Quantum Mechanics is not a complete theory.

The ideas in this paper have been highly influenced, however not determined, by the reading of [2].

\section{Main argument}

To motivate the discussion that follows, let's consider a numerous population of cubic dice. We perform an experiment consisting of rolling all dice at regular time intervals $n \delta t$, and recording the results. Let $p_{i}(0), i=1, \cdots, 6$ be the intial distribution of probabilities. As time transcurs, and the dice are repeatedly rolled, because of wear and tear, this distribution will slowly change. Thus we have six functions $p_{i}(n), i=1, \cdots, 6$. From this function we define a vector:

$$
\psi(n)=\left(\begin{array}{c}
\sqrt{p_{1}(n)} \\
\sqrt{p_{2}(n)} \\
\sqrt{p_{3}(n)} \\
\sqrt{p_{4}(n)} \\
\sqrt{p_{5}(n)} \\
\sqrt{p_{6}(n)}
\end{array}\right)
$$

where $n$ counts the time intervals, as explained before. It is clear that

$$
p_{i}(n)=\psi_{i}^{2}(n)
$$


Because of the normalization condition:

$$
\sum_{i=1}^{6} p_{i}(n)=1,
$$

though the vector $\psi$ changes, the scalar product $\langle\psi| \psi>$ remains constant, equal to 1 . As a consequence, for each value of $n$, there is an orthogonal transformation

$$
\mathcal{O}(n)=\mathcal{I}+\delta \mathcal{O}(n)
$$

such that

$$
\begin{gathered}
\psi(n+1)=\mathcal{O}(n) \psi(n), \text { or } \\
\psi(n+1)-\psi(n)=\delta \mathcal{O}(n) \psi(n)
\end{gathered}
$$

where $\delta \mathcal{O}(n)$ is an infinitesimal skew-symmetrical matrix-therefore, the generator of a one-dimensional group of rotations around a fixed axis in a sixdimensional space - that depends on $n$ and possibly on the population (the dice's material and other things). In the special case that $\mathcal{O}(n)$ is constant and $\delta t$ is small, we can replace (2) with a differential equation:

$$
\frac{d \psi}{d t}=\hat{o} \psi
$$

where $\hat{o}$ is a skew-symmetrical matrix or:

$$
i \frac{d \psi}{d t}=\hat{h} \psi
$$

where $\hat{h}$ is an hermitian matrix: $\hat{h}=i \hat{o}$.

Though the introduction of the imaginary unit $i$ is somewhat artificial in this case, we pass now to consider a situation, where it appears from a necessity.

Let the population be now a numerous collection of particles moving at random - in one dimension - and $\rho(x, t)$ be the corresponding density of probability. Though we might be tempted to define $\psi(x, y)=\sqrt{\rho(x, t)}$, as we did before, this is not advisable, because the function $\psi(x, t)$ might not be differentiable at the points where $\rho(x, t)$ is equal to zero, even if $\rho(x, t)$ is differentiable everywhere. Such is the case for a distribution of the form:

$$
\rho(x, 0)=\frac{1}{\pi} \frac{\sin ^{2} x}{x^{2}}
$$


because

$$
\sqrt{\rho(x, 0)}=\frac{1}{\sqrt{\pi}} \frac{|\sin x|}{|x|},
$$

which is not differentiable at the points where $\sin (x)=0: 0, \pm \pi, \pm 2 \pi, \cdots$. This difficulty is surmounted if we allow $\psi(x, t)$ to be a complex function, because in this way we can continuously pass from $\psi(x, t)=\sqrt{\rho(x, t)}$ at some point on the $x$ axis to $\psi(x, t)=-\sqrt{\rho(x, t)}$ at another point. So, we write $\rho=\psi^{\star} \psi$. This mathematical fact is disregarded in the de BroglieBohm formulation of Quantum Mechanics [3], which in our view is a serious deficiency of this theory, because it casts doubts on the validity of the concept of quantum potential, which is fundamental to it. See also $[4,5,6,7,8]$.

Like in the case of the dice, as time goes on $\psi(x, t)$ will change -in general-because $\rho(x, t)$ whill change also. However, because of the normalization condition, $\psi(x, t+\delta t)$ can be obtained from $\psi(x, t)$ as the result of an infinitesimal unitary transformation in the space of quadratically integrable complex functions of one real variable. Notice that we are not making any assumptions about how the motion of particles takes place, what they are, or if they follow continuous or differentiable paths. Our only assumption is that a particle must have a position at each instant.

To avoid unnecessary repetitions, from this point on, we will consider a distribution $\rho(x, y, z, t)$ in a tridimensional space, and other more general cases. The distinction should be clear from the context.

As we said before, because of the normalization condition, there is at every time an infinitesimal (linear) unitary transformation $\mathbf{I}-i \delta t \hat{h}$, such that:

$$
\begin{gathered}
\psi(x, y, z, t+\delta t)=(\mathbf{I}-i \delta t \hat{h}) \psi(x, y, z, t), \\
\psi^{\star}(x, y, z, t+\delta t)=\left(\mathbf{I}+i \delta t \hat{h}^{\star}\right) \psi^{\star}(x, y, z, t),
\end{gathered}
$$

and

$$
i \delta t \int_{\mathcal{R}^{3}} \hat{h}^{\star}\left(\psi^{\star}\right) \psi-\psi^{\star} \hat{h}(\psi) d^{3} \vec{x}=0
$$

Hypotesis We suppose that $\hat{h}$ is independent on the state of the system (now described by the wave function $\psi$ ) and also of time, by virtue of the PRINCIPLE OF HOMOGENITY OF TIME. As a consequence:

$$
\hat{h}=\hat{h}^{\dagger}
$$


must be a time independent hermitian operator. Our simplest choice being:

$$
\hat{h}=\frac{\kappa}{2} \Delta,
$$

where $\kappa$ is a real constant: Because Laplace's operator is hermitian and invariant in translations and rotations of the reference frame. This leads us to an equation:

$$
i \frac{\partial \psi}{\partial t}=\frac{\kappa}{2} \Delta \psi
$$

The average value of the coordinate $x_{i}$ can be obtained as:

$$
\left\langle X_{i}\right\rangle=\int_{\mathcal{R}^{3}} \psi^{\star} \hat{X}_{i} \psi d^{3} \vec{x}
$$

where $\hat{X}_{i}(\psi)=x_{i} \psi$. Also

$$
\begin{gathered}
\left\langle\dot{X}_{i}\right\rangle=\int_{\mathcal{R}^{3}}\left[\frac{\partial \psi^{\star}}{\partial t} \hat{X}_{i}(\psi)+\psi^{\star} \hat{X}_{i}\left(\frac{\partial \psi}{\partial t}\right)\right] d^{3} \vec{x} \\
=i \int_{\mathcal{R}^{3}} \hat{h}^{\star}\left(\psi^{\star}\right) \hat{X}_{i} \psi-\left(\psi^{\star}\right) \hat{X}_{i} \psi \hat{h}(\psi) d^{3} \vec{x} \\
\int_{\mathcal{R}^{3}} \psi^{\star} i\left[\hat{h} \hat{X}_{i}-\hat{X}_{i} \hat{h}\right] \psi d^{3} \vec{x}=\int_{\mathcal{R}^{3}} \psi^{\star} i \kappa \frac{\partial \psi}{\partial x_{i}} d^{3} \vec{x}
\end{gathered}
$$

as it can be verified from 4 . In the last equation we identify a velocity operator:

$$
\hat{\dot{X}}_{i}=i \kappa \frac{\partial}{\partial x_{i}}
$$

and, by an analogous process an acceleration operator:

$$
\hat{\ddot{X}}_{i}=i\left[\hat{h} \hat{\dot{X}}_{i}-\hat{\dot{X}}_{i} \hat{h}\right] \equiv 0,
$$

showing that this formalism is in agreement with the PRINCIPLE OF INERTIA at least in a statistical sense.

Let's consider now a population made of a numerous sample of twoparticle systems of the same kind-like hydrogen atoms. The distribution function will take the form:

$$
\rho=\rho\left(x_{1}, y_{1}, z_{1}, x_{2}, y_{2}, z_{2}, t\right) .
$$

We factor $\rho=\psi^{\star} \psi$ as before, and we find: 


$$
i \frac{\partial \psi}{\partial t}=\kappa_{1} \Delta_{1} \psi+\kappa_{2} \Delta_{2} \psi+\Omega\left(\left|\vec{r}_{1}-\vec{r}_{2}\right|\right) \psi
$$

In a similar fashion, the velocity operators are:

$$
\hat{\dot{X}}_{1}=i \kappa_{1} \frac{\partial}{\partial x_{1}}, \hat{\dot{X}}_{2}=i \kappa_{2} \frac{\partial}{\partial x_{2}}, \cdots
$$

When we pass to the accelerations we get:

$$
\hat{\ddot{X}}_{1}=\kappa_{1} \frac{\partial \Omega}{\partial x_{1}}, \hat{\ddot{X}}_{1}=\kappa_{2} \frac{\partial \Omega}{\partial x_{2}}, \cdots
$$

suggesting that $\kappa_{i}$ is negative and inversely proportional to the mass of each particle, BECAUSE of NEWTON's LAWS AND THE PRINCIPLE OF CORRESPONDENCE. Thus we come to the equation:

$$
i \hbar \frac{\partial \psi}{\partial t}=-\frac{\hbar^{2}}{2 m_{1}} \Delta_{1} \psi-\frac{\hbar^{2}}{2 m_{2}} \Delta_{2} \psi+V\left(\left|\vec{r}_{2}-\vec{r}_{1}\right|\right) \psi,(V=\hbar \Omega .)
$$

which is Schrödinger equation as we know it. Of course, $\hbar$ is a constant to be determined by experiment, as it has been determined.

It is apparent now, that all the information we can have about the state of an ensemble of a quantum systems is encoded in the wave function $\psi$, which is nothing but a mathematical artifact. This comes from our restriction to have $\hat{h}$ independent on the state of the ensemble. The actual operator, the hamiltonian, was derived more or less on the same grounds on which the distribution of velocities of an ideal gas was derived by Maxwell - the symmetries of space, the homogeneity of time, and Occam's Razor Principle - with no consideration whatsoever of the structure of the gas molecules. The difference is this: we have also appealed to Newton's Laws and the Principle of Correspondence. In our view, this reinforces the Einstein-Podolsy-Rosen presumption that Quantum Mechanics is not a complete theory $[9,10]$.

From this point on, the development of quantum ideas can follow the same path as in any standard texbook, on theoretical physics like [11].

One more thing we want to mention, regarding the de Broglie-Bohm formulation of Quantum Mechanics. From equation (4) now written as ${ }^{1}$ :

$$
i \hbar \frac{\partial \psi}{\partial t}=-\frac{\hbar^{2}}{2 m} \Delta \Psi
$$

\footnotetext{
${ }^{1}$ We do not include a potential energy, because the presence of a potential energy implies an interaction, therefore the system is not closed and, as a consequence, the hamiltonian must be time dependent. This is a consideration which is frequently disregarded.
} 
it is not difficult to prove the continuity equation:

$$
\frac{\partial \rho}{\partial t}+\nabla \cdot\left(\frac{\rho \nabla S}{m}\right)=0
$$

where $S$ is the phase of the wave function. From this equation, the proponents of the de Broglie-Bohm theory draw the conclussion that the velocity of quantum particles is [3, p. 79 eq. 3.2.19]

$$
\vec{v}(\vec{x}, t)=1 / m \nabla S .
$$

The fact is that equation (7) does not completely determine the current of probability, but only its divergence: the vortex remains unknown. That's why in the base state of hydrogen, for example, they are forced to conclude that $\vec{r}=\vec{r}_{e}-\vec{r}_{p}$ remains constant, because the phase of a real wave function is independent of $\vec{r}$.

\section{Conclussions and remarks}

As we mentioned before, we have not made any assumptions about how the motion of particles takes place, what they are, or if they follow continuous or differentiable paths. Our only assumption was that a particle must have a position at each instant. It is because of this and other reasons we have already exposed, that we conclude that the wave function is just a mathematical artifact and quantum mechanics is not a complete theory. If we are right, by no means that implies that we can come out with something better, because of our natural limitations. After all, we are part of the universe we want to understand.

The normalization condition that we have imposed on the distribution $\rho(x, t)$ implies that we consider non overlaping regions of space as mutually exclusive events, in the sense of the classical theory of probability. This means that a particle cannot be found inside two non overlaping regions at the same time, or simultaneously.

The concept of simultaneity has been challenged by the special theory of relativity, based on the negative result of Michelson's experiment and other facts, and as a consequence, by Einstein's theory of gravitation. This means that we might need to finish developing a theory of probabilities that is coherent with relativity when space-time relations are involved, as a first step towards a coherent unification of quantum mechanics and relativity [12, See for example ] 


\section{Acknowledgements}

The author wishes to thank Prof. Charles Francis, from Jesus College Cambridge, for invaluable discussions through researchgate.org as well as M. A. Bruce Braley and M. S. Joel Roberto Chavoya for their kind disposition to proof read and suggest corrections to improve the readability of this paper.

\section{References}

[1] R. P. Feynman; QED The strange theory of light and matter; Princeton Science Library (1988) p. 9.

[2] C. Francis; The Hilbert space of conditional clauses; arXiv:1205.4607v2 [physics.gen-ph].

[3] P. R. Holland; The Quantum Theory of Motion; Cambridge University Press (2000); p. 69 eqs. 3.2 .4 and 3.2.6 \& p. 72 eq. 3.2.16.

[4] E. Madelung; Quantentheorie in hydrodynamischer form; Z. Phys. 40 (3-4): 322-326 (1927).

[5] O. Chavoya-Aceves; A de Broglie-Bohm-like model for Klein-Gordon equation; Il Nuovo Cimento B, vol. 118, Issue 06, p. 569 (2003).

[6] O. Chavoya-Aceves; A de Broglie-Bohm-like model for Dirac equation; Il Nuovo cimento. B, Vol.118 No.9, (2003).

[7] O. Chavoya-Aceves; Generalization of Hamilton-Jacobi method and its consequences in classical, relativistic, and quantum mechanics; [arXiv:quant-ph/0409012].

[8] R. Tsekov; Bohmian mechanics versus Madelung quantum hydrodynamics; Ann. Univ. Sofia, Fac. Phys. Special Ed. (2012) pp. 112-119 [arXiv0904.0723]; eqs. 4, 6, 9, and 10.

[9] A. Einstein, B. Podolsky, N. Rosen; Can Quantum-Mechanical Description of Physical Reality be Considered Complete?; Phys. Rev. 47 777780 (1935).

[10] O. Chavoya-Aceves; Logical refutation of the Einstein-Podolsky-Rosen argument; Physics Essays V. 26: pp. 21-23, (2013). 
[11] L. D. Landau \& E. M. Lifshits; Quantum Mechanics: Non-relativistic Theory; Butterworth-Heinemann (1977).

[12] S. Saunders; Space-Time and Probability; Chance in Physics: Foundations and Perspectives, J. Bricmont, D. Dürr, M.C. Galavotti, G. Ghirardi, F. Petruccione, N. Zanghi (eds.), Springer-Verlag, 2001. 